\title{
Rumor evolution in social networks
}

\author{
Yichao Zhang, ${ }^{1,2}$ Shi Zhou, ${ }^{2, *}$ Zhongzhi Zhang, ${ }^{3,4, \dagger}$ Jihong Guan, ${ }^{1, \ddagger}$ and Shuigeng Zhou ${ }^{3,4, \S}$ \\ ${ }^{1}$ Department of Computer Science and Technology, Tongji University, 4800 Cao' an Road, Shanghai 201804, China \\ ${ }^{2}$ Department of Computer Science, University College London, Gower Street, London WC1E 6BT, United Kingdom \\ ${ }^{3}$ School of Computer Science, Fudan University, Shanghai 200433, China \\ ${ }^{4}$ Shanghai Key Lab of Intelligent Information Processing, Fudan University, Shanghai 200433, China
}

(Received 12 June 2012; revised manuscript received 23 October 2012; published 15 March 2013)

\begin{abstract}
The social network is a main tunnel of rumor spreading. Previous studies concentrated on a static rumor spreading. The content of the rumor is invariable during the whole spreading process. Indeed, the rumor evolves constantly in its spreading process, which grows shorter, more concise, more easily grasped, and told. In an early psychological experiment, researchers found about $70 \%$ of details in a rumor were lost in the first six mouth-to-mouth transmissions. Based on these observations, we investigate rumor spreading on social networks, where the content of the rumor is modified by the individuals with a certain probability. In the scenario, they have two choices, to forward or to modify. As a forwarder, an individual disseminates the rumor directly to their neighbors. As a modifier, conversely, an individual revises the rumor before spreading it out. When the rumor spreads on the social networks, for instance, scale-free networks and small-world networks, the majority of individuals actually are infected by the multirevised version of the rumor, if the modifiers dominate the networks. The individuals with more social connections have a higher probability to receive the original rumor. Our observation indicates that the original rumor may lose its influence in the spreading process. Similarly, a true information may turn out to be a rumor as well. Our result suggests the rumor evolution should not be a negligible question, which may provide a better understanding of the generation and destruction of a rumor.
\end{abstract}

DOI: 10.1103/PhysRevE.87.032133

PACS number(s): 02.50.Le, 89.75.Fb, 05.70.Jk

\section{INTRODUCTION}

Rumor spreading is a fundamental topic in psychology [1] and sociology [2]. In the past decade, rumor spreading on social networks, e.g., small-world networks [3-5] and scale-free networks [5-8], has attracted a lot of attention from physical and sociological communities [8-13]. In the small-world network, a threshold $P_{c}$ of spreading in the rewiring probability of links was reported. As the probability is larger than $P_{c}$, i.e., when the average path length of the network is short enough, rumors can be disseminated globally. On the other hand, in the scale-free networks, the discussions are mainly focused on the spreading efficiency and the final infected ratio, which was analytically solved by a mean-field approximation [6]. Most recently, a model describing two propagating rumors with different probabilities of acceptance is also worth a mention [14].

Simultaneously, the development of the dynamical model itself is also pretty heuristic $[15,16]$. Some researchers took into consideration the accumulation effect of rumor in the process of persuading the ignorant [15] and others investigated the degeneration of information on a spatial system [16]. Both of these works provide valuable insight into the rumor dynamics.

In real social networks, the rumor spreading process is much more complicated than the reported scenarios [17-21]. The rumor evolves constantly in its spreading process, which grows shorter, more concise, more easily grasped, and told [1]. The behavior originates from the cumulative modifications during

\footnotetext{
*s.zhou@cs.ucl.ac.uk

${ }^{\dagger}$ zhangzz@fudan.edu.cn

${ }^{\ddagger}$ jhguan@ tongji.edu.cn

§sgzhou@fudan.edu.cn
}

the spreading process, which is called "Chinese Whispers" or "Telephone" in some conditions [22]. The phenomenon is easily observed in real social networks. For example, on a microblogging site Twitter, once a user discusses a certain topic, his or her followers will understand the topic indirectly. If it is a rumor, the following discussions can roughly be classified as affirmative, negative, curious, unrelated, and unknown arguments [23]. No matter which class the preceeding tweets belong to, they are still the variants of the original rumor. Thus, whether the original rumor can infect the whole network depends not only on the existence of the connections among individuals, but also on their strategies. We divide the strategies into two classes, to forward it directly or to change it before spreading it out. For convenience, the individuals forwarding the rumor are denoted as forwarders. Conversely, the individuals changing the rumor are denoted as modifiers. In real email systems, modifiers can be not only users but also machines, called remailers [24].

In this paper, we will investigate rumor spreading in social networks, where individuals have two static behaviors, to forward and to modify. As long as they are receiving a rumor, a forwarder will deliver it to its neighbors directly, while an ignorant modifier will deliver the rumor after revising it once. When spreading in a network including modifiers, the rumor probably has to experience a series of revisions. Even if the original rumor is an unconfirmed truth, the spreading process may turn it into a rumor as well. Thus, this general model may draw a relatively complete picture of real rumor spreading, including the generation and destruction of a rumor. Previous works taking all the individuals as forwarders [3-8] is a special case of law. In previous models [3-8], the spreading rate and the annihilation rate are two key parameters, which govern the spreading process and the final infected ratio. In this paper, they are not of concern. Thus, we set both of them to focus 
on the evolution of the rumor itself. Given that the evolution of rumor content is a sociological topic, we only focus on the revised frequency here.

\section{RUMOR EVOLUTION ON SOCIAL NETWORKS}

As discussed in the original rumor model (D. J. Daley and D. G. Kendall model) [9], individuals can play three roles: ignorants, spreaders, and stiflers, whose densities are denoted by $i(t), s_{x}(t)$, and $r(t)$, respectively. Here, $s_{x}$ denotes the version $x(x=1,2, \ldots, n)$ of a rumor. The original version is version 1 . We set the normalization condition $i(t)+s_{\text {sum }}(t)+$ $r(t)=1$ and $s_{\text {sum }}=\Sigma_{x} s_{x}$. The role of an individual starts with an ignorant. If this individual is infected before the rumor vanishes, they will turn to be a spreader. Finally, they will be a stifler once they spread the rumor to a spreader or stifler. If this individual is not infected during the process, they will keep their role. Besides these dynamical roles, each individual also has two types of static behaviors, to forward or to modify. The behaviors of individuals are fixed in the spreading process. We will discuss all the possible cases in what follows.

When receiving a certain version of the rumor, an ignorant forwarder becomes a spreader of the version. When receiving two or more different versions of the rumor, the forwarder accepts the latest version. For example, given $a>b$, if the forwarder receives the versions $a$ and $b$ at the same step, their role becomes $S_{a}$.

Once receiving the rumor, an ignorant modifier revises it before disseminating it out. When receiving two or more different versions of the rumor at the same step, the modifier revises the latest version. For example, given $a>b$, if the modifier receives versions $a$ and $b$ at the same step their role becomes $S_{a+1}$.

If a spreader receives the original rumor or the revised versions in the following steps, they will turn to be a stifler, no matter whether they are a forwarder or modifier. This is simply because once the spreader has disseminated similar information to their neighbors, there is no need to do that again [5]. On the other hand, the neighbors who send the versions of the rumor to them would not be interested in the similar information as well. They will turn out to be stiflers at the same step. To explain this point more clearly a simple illustration is shown in Fig. 1.

\section{A. Rumor evolution on scale-free networks}

When a rumor is injected into heterogeneous networks such as A. L. Barabási and R. Albert networks [25], the set of coupled properties can be written as the following. Consider an ignorant forwarder $i$ with degree (or connectivity) $k$ after $t$ steps. When receiving the rumor $x$, a probability with which it becomes a spreader $s_{x}$ is

$$
P_{i \rightarrow s_{x}}^{i s}(t, k)=F k P(k) i_{k}(t) \Sigma_{k^{\prime}} \frac{k^{\prime} P\left(k^{\prime}\right) s_{k^{\prime}}(t)}{\langle k\rangle},
$$

where $P(k)$ denotes the degree distribution of the networks and $F$ denotes the forwarders' fraction. If $i$ is an ignorant modifier, the probability with which it becomes a revised rumor spreader $s_{x+1}$ is

$$
P_{i \rightarrow s_{x+1}}^{i s}(t, k)=(1-F) k P(k) i_{k}(t) \Sigma_{k^{\prime}} \frac{k^{\prime} P\left(k^{\prime}\right) s_{k^{\prime}}(t)}{\langle k\rangle} .
$$
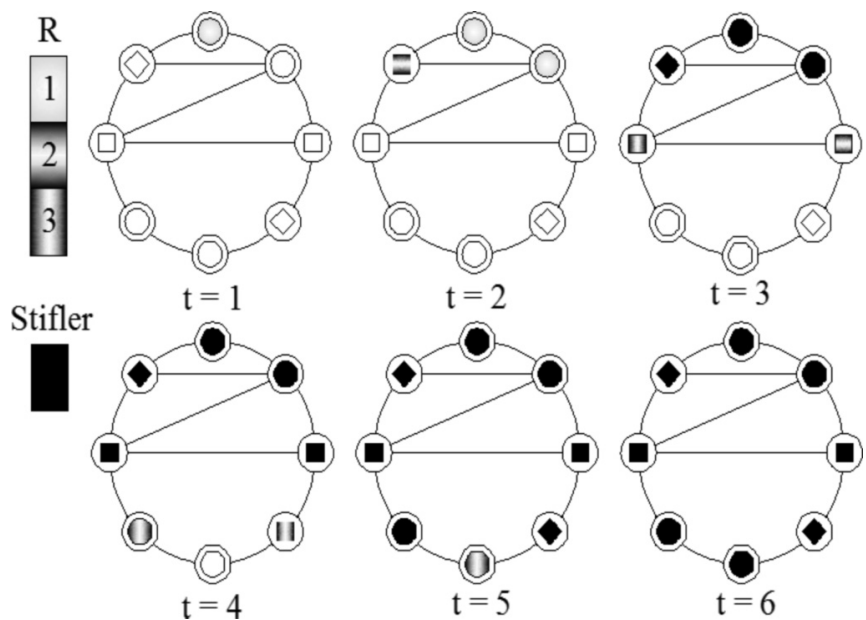

FIG. 1. Illustration of rumor spreading in a network with forwarders and modifiers. Circles denote the forwarders and squares denote the modifiers. We set a rumor start with light gray, whose hatch changes once when encountering a modifier. $R$ denotes the revised frequency of the rumor. The original rumor is denoted by $R=1$. Solid black denotes stifler. The illustration represents the whole process of rumor spreading in six time steps.

The probability with which a spreader $s_{x}$ becomes a stifler $r$ is

$$
P_{s_{x} \rightarrow r}^{s r}(t, k)=k P(k) s_{k}(t) \Sigma_{k^{\prime}} \frac{k^{\prime} P\left(k^{\prime}\right)\left[s_{k^{\prime}}(t)+r_{k^{\prime}}(t)\right]}{\langle k\rangle} .
$$

We define

$$
\left\langle R_{k}\right\rangle=\frac{\sum_{i \in\{i \mid \operatorname{degree}(i)=k\}} i_{R}}{N_{k}},
$$

where $i_{R}$ denotes the last version of the rumor at individual $i$ before individual $i$ turns out to be a stifler, where $i=$ $1,2, \ldots, N$ and $R=1,2, \ldots\left\langle\left\langle R_{k}\right\rangle\right.$ represents the frequency that a rumor has been revised on average before annihilating at an individual with degree $k$. The rate equation for the average revised frequency $\left\langle R_{k}\right\rangle$ on degree $k$ can be written as

$\frac{d\left\langle R_{k}(t)\right\rangle}{d t}=(1-F) P(k) k i_{k}(t) \Sigma_{k^{\prime}} \frac{k^{\prime} P\left(k^{\prime}\right) s_{k^{\prime}}(t)\left\langle R_{k^{\prime}}(t)\right\rangle}{\langle k\rangle}$.

The evolution of the densities $s_{k}(t)$ and $r_{k}(t)$ satisfy the following set of coupled differential equations:

$$
\begin{gathered}
\frac{d i_{k}(t)}{d t}=-k P(k) i_{k}(t) \Sigma_{k^{\prime}} \frac{k^{\prime} P\left(k^{\prime}\right) s_{k^{\prime}}(t)}{\langle k\rangle}, \\
\frac{d s_{k}(t)}{d t}=k P(k) i_{k}(t) \Sigma_{k^{\prime}} \frac{k^{\prime} P\left(k^{\prime}\right) s_{k^{\prime}}(t)}{\langle k\rangle} \\
-k P(k) s_{k}(t) \Sigma_{k^{\prime}} \frac{k^{\prime} P\left(k^{\prime}\right)\left[s_{k^{\prime}}(t)+r_{k^{\prime}}(t)\right]}{\langle k\rangle}, \\
\frac{d r_{k}(t)}{d t}=k P(k) s_{k}(t) \Sigma_{k^{\prime}} \frac{k^{\prime} P\left(k^{\prime}\right)\left[s_{k^{\prime}}(t)+r_{k^{\prime}}(t)\right]}{\langle k\rangle} .
\end{gathered}
$$

\section{B. Rumor evolution on small-world networks}

When a rumor is injected into the homogeneous networks such as D. J. Watts and S. H. Strogatz networks [26], the set of coupled properties can be written as what follows. Consider an ignorant forwarder $i$ after $t$ steps. When receiving the rumor 
$x$, the probability with which it becomes a spreader $s_{x}$ is

$$
P_{i \rightarrow s_{x}}^{i s}(t)=F\langle k\rangle i(t) s(t) .
$$

If $i$ is an ignorant modifier, the probability with which it becomes a revised rumor spreader $s_{x+1}$ is

$$
P_{i \rightarrow s_{x+1}}^{i s}(t)=(1-F)\langle k\rangle i(t) s(t) .
$$

The probability with which a spreader $s_{x}$ becomes a stifler $r$ is

$$
P_{s_{x} \rightarrow r}^{s r}(t)=\langle k\rangle s(t)[s(t)+r(t)] .
$$

Thus, in this case, the rate equation for the average revision frequency $R(t)$ can be written as

$$
\frac{d\langle R(t)\rangle}{d t}=(1-F)\langle k\rangle i(t) s(t)\langle R(t)\rangle,
$$

where the evolution of the densities $s(t)$ and $r(t)$ satisfy the following set of coupled differential equations:

$$
\begin{gathered}
\frac{d i(t)}{d t}=-\langle k\rangle i(t) s(t), \\
\frac{d s(t)}{d t}=\langle k\rangle s(t)\{i(t)-[s(t)+r(t)]\}, \\
\frac{d r(t)}{d t}=\langle k\rangle s(t)[s(t)+r(t)] .
\end{gathered}
$$

In the infinite time limit, previous studies $[5,6]$ showed that the fraction of individuals infected by the rumor can be written as

$$
r(\infty)=1-e^{-2 r(\infty)} .
$$

In this scenario, the ratio is a constant, which is only determined by Eqs. (13) and (15) and the normalization condition $i(\infty)+r(\infty)=1$ for $s_{\text {sum }}(\infty)=0$. More to the point, one can find that the final infected ratio $r(\infty)$ is irrelevant to the evolution of the rumor. For the evolution, the system of differential equations Eq. (12) and (13) can be analytically solved. With $\langle R(0)\rangle=\left(\frac{F}{N}+\frac{2(1-F)}{N}\right)$, one can derive

$$
\langle R(t)\rangle=\exp \left[(F-1) i(t)+(1-F) \frac{N-1}{N}-\ln \frac{2-F}{N}\right] .
$$

In the infinite time limit, we have

$$
\begin{aligned}
\langle R(\infty)\rangle= & \exp \left\{(F-1)[1-r(\infty)]+(1-F) \frac{N-1}{N}\right. \\
& \left.-\ln \frac{2-F}{N}\right\} .
\end{aligned}
$$

Considering $r(\infty)$ is a constant, $\langle R(\infty)\rangle$ only depends on the forwarders' fraction $F$ and the total number of individuals $N$ for the WS networks.

\section{DISTRIBUTIONS OF REVISED FREQUENCIES}

To clarify the result of evolution, we then run extensive simulations on both the WS and BA networks for five different values of $F$. Also, we investigate the effects of totally regular and random structures. We generate ten WS networks, BA networks, regular graphs (networks), and random graphs (networks) using random seeds. In Fig. 2, we measure the distributions of $R$. We define $\Phi(R)$ as the number of individuals who were the spreaders of the rumor revised $R$ times before the rumor vanishes.
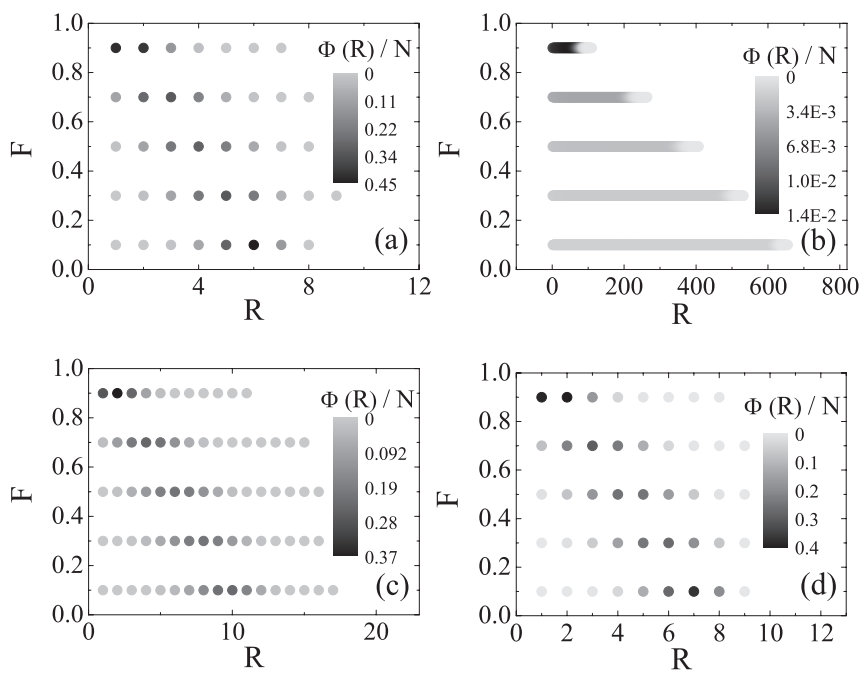

FIG. 2. $\Phi(R) / N$ as a function of $R$ and $F$ for different topologies with $F=0.1,0.3,0.5,0.7,0.9 . N$ denotes the size of the networks, which is 4096 in our simulations. Panel (a) shows the simulation results obtained on BA scale-free networks, which are generated by $m_{0}=m=3$ [25]. Panel (b) shows the simulation results obtained on regular graphs, which are formed by 4096 identical individuals of degree 6. Panel (c) shows the simulation results obtained on WS small-world networks, which are generated by randomly rewiring $10 \%$ of the links in the regular graphs. Panel (d) shows the simulation results obtained on random graphs, which are generated by randomly rewiring all the links in the regular graphs [26]. With the initial conditions $i(0)=\frac{N-1}{N}, s(0)=\frac{1}{N}$, and $r(0)=0$, simulation results were obtained by ten random assignments of modifiers on ten different realizations of the same type of network specified by the appropriate parameters. Each plot in this figure corresponds to $100 * N_{k}(k=6)$ simulations. For each run, we set a randomly picked individual with degree 6 as the first spreader.

As shown in Figs. 2(a), 2(c), and 2(d), for the BA networks, one can find out the position of the maximum of the distribution of $R$ shifts to the left with $F$. Simultaneously, all the maximums grow with $F$. In Fig. 2(b), for the regular graphs, one can observe that the distribution is relatively uniform. The positions of the maximum shift to the left with $F$ as well. Contrarily, the versions of the rumor are much richer. One can observe that the maximum grows with $F$, but the growth is relatively limited.

For $F$ close to 0 , the distributions of $R$ on all the networks tend to reach a relatively uniform status. In Fig. 2(b) one can observe that the number of individuals infected by various versions are basically identical for regular graphs. In Figs. 2(a), 2(c), and 2(d), one can observe that the majority of the individuals are infected by the versions revised more than our times. For $F$ close to 1, Figs. 2(a)-2(d) show that the majority of the individuals are infected by the versions revised less than three times. This observation indicates that the original rumor can keep its influence on the individuals only when most of them are forwarders in the social networks.

To clarify the relation between the revised frequency and the topological features of networks, we measure the average revised frequency $\left\langle R_{k}\right\rangle$ for the individuals with degree $k$. As shown in Fig. 3(a), one can observe that the individuals with low degree have a relatively high $\left\langle R_{k}\right\rangle$ in the BA networks. 

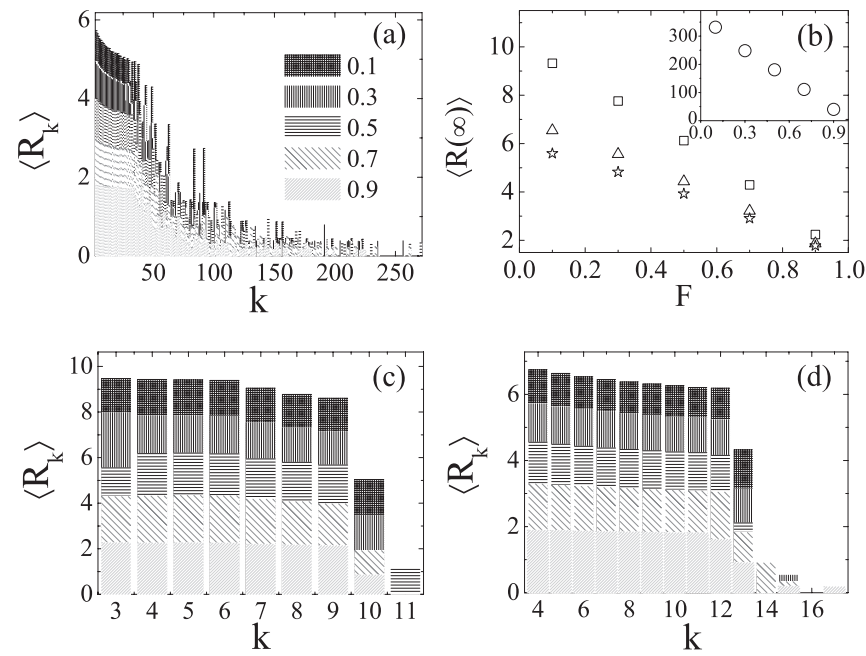

FIG. 3. $\left\langle R_{k}\right\rangle$ as a function of individuals' degree $k$ for different topologies with $F=0.1,0.3,0.5,0.7,0.9$. Panels (a), (c), and (d) show simulation results for BA scale-free networks, WS small-world networks, and random graphs. Panel (b) shows the relations between $\langle R(\infty)\rangle$ and $F$ for regular graphs (circles in the inset), WS networks (squares), random graphs (triangles), and BA networks (stars). Panels (c) and (d) share the same legend of $F$ with panel (a).

The feature decays with $F$. For the regular graphs, all the individuals' degrees are 6 . Hence, we only show the relation between $\langle R(\infty)\rangle$ and $F$ in Fig. 3(b). In Fig. 3(b), one can observe that $\langle R\rangle$ decays with $F$ for all the topologies investigated in this paper. The inset shows the relation between $\left\langle R_{6}(\infty)\right\rangle$ and $F$ for the regular graphs. Obviously, The speed of decay for the regular graphs is much higher than that of the others. In Figs. 3(c) and 3(d), for the WS networks and random graphs, one can observe the distribution of $\left\langle R_{k}\right\rangle$ is close to a uniform distribution for the individuals with small degree. For the BA scale-free networks, $\left\langle R_{k}\right\rangle$ is directly proportional to $N_{k}$. As a function of $k,\left\langle R_{k}\right\rangle$ follows a power law. Interestingly, for the WS small-world networks and random graphs, the distribution of $\left\langle R_{k}\right\rangle$ is irrelevant to the degree distribution generally. The similarity of all the irregular networks investigated here is that the individuals with a higher connectivity have a higher probability to receive the less-revised rumor.

\section{CONCLUSION}

To sum up, rumor as a common social phenomenon has been investigated in various topological models. In previous studies, the content of a rumor was set to be invariable in its spreading process. Indeed, most rumors evolve constantly, which may grow shorter, more concise, more easily grasped, and told. They may also be commented on or questioned. In this paper, we have proposed a rumor model in social networks, where two static behaviors of individuals, to modify or to forward, govern the evolution of the rumor. As defined in the previous models, each individual may have three dynamical roles, ignorant, spreader, and stifler. Initially, we inject a rumor into a network where all the individuals are ignorant. When an ignorant forwarder receives a rumor they become a spreader and spread the rumor to their neighbors directly. When an ignorant modifier receives a rumor they become a spreader as well. However, they revise the content before spreading. If they receive the rumor or the revised version again in the following steps they will turn out to be a stifler. This is because if they disseminated similar information to their neighbors before, the neighbors may lose interest in the information. When all the individuals are forwarders our model can be reduced to the previous rumor models.

We have run extensive simulations to investigate the distributions of the revised frequency $R$ on various topological structures. For the BA scale-free networks, regular graphs, WS small-world networks, and random graphs we found that the position of the maximum of the distributions shifts to the left with the fraction of forwarders $F$. For a small $F$, the distributions on all the networks tend to reach a relatively uniform status. The majority of the individuals are infected by the multirevised versions. For a large $F$, the original rumor can keep its influence on the individuals.

To clarify the relation between the revised frequency and topological structure, we have measured the average revised frequency $\left\langle R_{k}\right\rangle$ as a function of degree $k$. For the BA scale-free networks, $\left\langle R_{k}\right\rangle$ is directly proportional to the number of individuals with degree $k$. Instead, for the WS small-world networks and random graphs, $\left\langle R_{k}\right\rangle$ does not depend on degree distribution generally. For all the irregular networks investigated in this paper, the well-connected individuals have a higher probability to receive the original rumor. For the regular graphs, respecting that all the individuals have an identical degree, we have measured the relation between the final average revised frequency for all the individuals $\langle R(\infty)\rangle$ and $F$. We found $\langle R(\infty)\rangle$ decays dramatically with $F$ in this regular structure. The speed of decay for regular graphs is much higher than that in the other structures.

As a common social phenomenon rumor evolution has been highly accelerated by modern information networks. The evolving rumor model we propose in this paper can provide a more realistic framework for the future research of rumor dynamics. We believe our results may provide a better understanding of rumor spreading in real social networks. Our observations are also capable of promoting related studies on agent-based rumor spreading.

\section{ACKNOWLEDGMENTS}

This research was supported by the National Natural Science Foundation of China under Grants No. 61173118, No. 61074119, No. 860873040, and No. 11275049, and 863 program under Grant No. 2009AA01Z135. J. Guan was also supported by the "Shuguang Scholar" Program of Shanghai Education Development Foundation under Grant No. 09SG23. Shi Zhou is supported by the UK Royal Academy of Engineering and the Engineering and Physical Sciences Research Council (EPSRC) under Grant No. 10216/70.
[1] G. W. Allport and L. Postman, The Psychology of Rumor (Russell and Russell, New York, 1947).
[2] S. C. Pendleton, Language Communication 1, 69 (1998).

[3] D. H. Zanette, Phys. Rev. E 64, 050901 (2001). 
[4] D. H. Zanette, Phys. Rev. E 65, 041908 (2002).

[5] Y. Moreno, M. Nekovee, and A. F. Pacheco, Phys. Rev. E 69, 066130 (2004).

[6] M. Nekovee, Y. Moreno, G. Bianconi, and M. Marsili, Physica A 374, 457 (2007).

[7] Y. Moreno, M. Nekovee, and A. Vespignani, Phys. Rev. E 69 , 055101 (2004).

[8] P. G. Lind, L. R. da Silva, J. J. S. Andrade, and H. J. Herrmann, Phys. Rev. E 76, 036117 (2007).

[9] D. J. Daley and D. G. Kendall, Nature (London) 204, 1118 (1964).

[10] P. G. Lind, L. R. da Silva, J. J. S. Andrade, and H. J. Herrmann, Europhys. Lett. 78, 68005 (2007).

[11] Ph. Blanchard, A. Krueger, T. Krueger, and P. Martin, arXiv:physics/0505031 [physics.soc-ph].

[12] J. L. Iribarren and E. Moro, Phys. Rev. Lett. 103, 038702 (2009).

[13] A. Grabowski, N. Kruszewska, and R. A. Kosiński, Phys. Rev. E 78, 066110 (2008).
[14] D. Trpevski, W. K. S. Tang, and L. Kocarev, Phys. Rev. E 81, 056102 (2010).

[15] P. S. Dodds and D. J. Watts, Phys. Rev. Lett. 92, 218701 (2004).

[16] E. Agliari, R. Burioni, D. Cassi, and F. M. Neri, Phys. Rev. E 73, 046138 (2006)

[17] A. Rapoport, Bull. Math. Biophys. 15, 523 (1953).

[18] B. Allen, J. Math. Sociol. 8, 265 (1982).

[19] S. P. Borgatti, Soc. Networks 27, 55 (2005).

[20] Z. Liu, J. Luo, and C. Shao, Phys. Rev. E 64, 046134 (2001).

[21] L. López and M. A. F. Sanjuán, Phys. Rev. E 65, 036107 (2002).

[22] S. Blackmore, The Meme Machine (Oxford University Press, New York, 2000).

[23] M. Mendozay, B. Pobletey, and C. Castillo, in Proceedings of First Workshop on Social Media Analytics (ACM, New York, 2010), p. 71

[24] G. F. du Pont, Journal of Technology Law \& Policy 6, 175 (2001).

[25] A. L. Barabási and R. Albert, Science 286, 509 (1999).

[26] D. J. Watts and S. H. Strogatz, Nature (London) 393, 440 (1998). 